\title{
Dissociable Roles for the Nucleus Accumbens Core and Shell in Regulating Set Shifting
}

\author{
Stan B. Floresco, Sarvin Ghods-Sharifi, Claudia Vexelman, and Orsolya Magyar \\ Department of Psychology and Brain Research Center, University of British Columbia, Vancouver, British Columbia, Canada V6T 1Z4
}

The ability to behave in a flexible manner is an executive function mediated in part by different regions of the prefrontal cortex. The present study investigated the role of two major efferents of the prefrontal cortex, the nucleus accumbens (NAc) core and shell, in behavioral flexibility using a maze-based strategy set-shifting task. During initial discrimination training, rats learned to use either an egocentric response or a visual-cue discrimination strategy to obtain food reward. During the set shift, animals had to shift from the previously acquired response or visual-cue-based strategy and learn the alternate discrimination. Inactivation of the NAc core, induced by infusion of the GABA agonists baclofen and muscimol, did not impair initial acquisition of either a response or visual-cue discrimination but severely disrupted shifting from one strategy to another. Analysis of the type of errors revealed that impairments in set shifting were not attributable to increased perseveration but to a disruption of the acquisition and maintenance of a new strategy. In contrast, inactivation of the NAc shell did not impair acquisition of either a response or a visual-cue discrimination, or shifting from one strategy to another. However, inactivation of the NAc shell before initial discrimination training improved performance during the set shift relative to control animals. These data indicate that the NAc core and shell make dissociable contributions to behavioral flexibility during set shifting. The NAc core facilitates the acquisition and maintenance of novel behavioral strategies and elimination of inappropriate response options, whereas the shell may mediate learning about irrelevant stimuli.

Key words: cognitive flexibility; ventral striatum; prefrontal cortex; associative learning; schizophrenia; GABA; rat

\section{Introduction}

The ability to behave flexibly in response to changing environmental contingencies is a survival skill mediated by the prefrontal cortex (PFC). Damage to subregions of the PFC impairs performance on tasks requiring animals to behave in a flexible manner (Dias et al., 1997; Brown and Bowman, 2002). For example, manipulations of the medial but not orbital PFC in rats impair attentional set shifting, entailing suppression of a previously relevant discrimination strategy and engagement of a novel one (Ragozzino et al., 1999; Birrell and Brown, 2000; Stefani et al., 2003; Floresco et al., 2006). In contrast, orbital but not medial PFC lesions impair reversal learning (Birrell and Brown, 2000; Chudasama et al., 2001; McAlonan and Brown, 2003).

A contemporary theory on frontal lobe function suggests that the "PFC allows for... flexibility by dynamically establishing task-relevant neural pathways in other brain systems" (Miller, 2000), allowing for a smooth transition from one cognitive strategy to another. One system that may interact with the PFC to

Received 0ct. 17, 2005; revised Jan. 23, 2006; accepted Jan. 23, 2006.

This work was supported by a Discovery Grant from the Natural Science and Engineering Research Council of Canada and a Young Investigator Award from the National Alliance for Research on Schizophrenia and Depression (S.B.F.). S.B.F. is a Canadian Institutes of Health Research New Investigator and a Michael Smith Scholar. We thank Richard Allison and Maric Tse for their assistance with behavioral testing, and we are grateful to Annie Block for her useful comments on this manuscript.

Correspondence should be addressed to Dr. Stan B. Floresco, Department of Psychology and Brain Research Center, University of British Columbia, 2136 West Mall, Vancouver, British Columbia, Canada V6T 1Z4. E-mail: floresco@psych.ubc.ca.

DOI:10.1523/JNEUROSCI.4431-05.2006

Copyright $\odot 2006$ Society for Neuroscience $\quad$ 0270-6474/06/262449-09\$15.00/0 mediate this executive function includes the dorsal and ventral striatum. Damage to the dorsal striatum in animals or patients with Parkinson's or Huntington's disease is associated with impairments in both reversal learning and set shifting (Owen et al., 1993; Lawrence et al., 1999; Ragozzino et al., 2002; Palencia and Ragozzino, 2005). Unlike PFC lesions, damage to the dorsomedial striatum impairs learning and maintenance of new strategies (Ragozzino et al., 2002), suggesting that these regions each mediate distinct processes that facilitate shifting from one strategy to another.

The core and shell regions of the nucleus accumbens (NAc) receive converging limbic and cortical input from the medial and orbital PFC, with the medial PFC projecting to both the core and shell and orbital projections terminating primarily in the core (Brog et al., 1993; Reynolds and Zahm, 2005). Thus, these nuclei are in an ideal anatomical position to regulate the control that multiple brain regions exert over behavior, leading to the hypothesis that the NAc contributes to determining response priorities under changing environmental conditions (Pennartz et al., 1994; Floresco et al., 2001). The NAc has been implicated in simpler forms of behavioral flexibility, including extinction (Annett et al., 1989; Reading and Dunnett, 1991) and behavioral switching (van den Bos et al., 1992). However, large NAc lesions do not disrupt visual, motor, or odor reversals (Stern and Passingham, 1995; Schoenbaum and Setlow, 2003) and cause variable effects on spatial reversals (Annett et al., 1989; Burk and Mair, 2001). In contrast, lesions of the NAc do impair behavior when rats must shift from a matching to nonmatching rule on a delayed response task 
mediated by the medial PFC (Dunnett, 1990; Reading and Dunnett, 1991). Thus, different subregions of the NAc may play a more prominent role in situations requiring shifts between incompatible behavioral strategies, or when an organism must attend to previously irrelevant stimuli to obtain reward. To investigate these issues further, we assessed the effects of inactivation of the NAc core and shell on performance of a strategy setshifting task mediated by the medial PFC and the dorsomedial striatum.

\section{Materials and Methods Apparatus}

Two identical four-arm cross mazes were used. The mazes were made of $1.5-\mathrm{cm}$-thick plywood and painted white. Each arm was $60 \mathrm{~cm}$ long and $10 \mathrm{~cm}$ wide, with 20-cm-high walls on each arm and with cylindrical food wells ( $2 \mathrm{~cm}$ wide $\times 1 \mathrm{~cm}$ deep) drilled into the end of each of the arms, 2 $\mathrm{cm}$ from the end wall. Four removable table legs attached to the ends of each arm elevated the mazes $70 \mathrm{~cm}$ above the floor. Removable pieces of white opaque plastic $(20 \times 10 \mathrm{~cm})$ were used to block the arms of the maze to form a " $\mathrm{T}$ " configuration. One maze resided in a $3 \times 3 \mathrm{~m}$ room, and the other resided in a room measuring $3.4 \times 3.4 \mathrm{~m}$. An individual rat was assigned to one particular testing room throughout the course of habituation and testing.

\section{Subjects and surgery}

Long-Evans rats (300-400 g; Charles River, Montreal, Quebec, Canada) were anesthetized with $100 \mathrm{mg} / \mathrm{kg}$ ketamine hydrochloride and $7 \mathrm{mg} / \mathrm{kg}$ xylazine and implanted with bilateral 23 gauge stainless steel guide cannulas into either the NAc core [flat skull: anteroposterior (AP), +1.6 $\mathrm{mm}$; mediolateral (ML), $\pm 1.8 \mathrm{~mm}$ from bregma; dorsoventral (DV), $-6.8 \mathrm{~mm}$ from dura] or the NAc shell [flat skull: AP, $+1.3 \mathrm{~mm}$; ML, $\pm 1.0 \mathrm{~mm}$ from bregma; DV, $-6.2 \mathrm{~mm}$ from dura]. Thirty-gauge obdurators flush with the end of the guide cannulas remained in place until the injections were made. Each rat was given at least $7 \mathrm{~d}$ to recover from surgery before training. During this recovery period, animals were food restricted to $85 \%$ of their free-feeding weight and were handled for at least $5 \mathrm{~min}$ per day.

\section{Habituation procedure}

The habituation procedure has been described previously (Ragozzino, 2002; Floresco et al., 2006). On the day before their initial exposure to the maze environment, rats were given $\sim 30$ sweetened food reward pellets (Bioserv, Frenchtown, NJ) in a glass dish placed in their home cages, which was removed once all of the pellets were eaten. On the first day of habituation, five reward pellets were placed in each of the arms of the maze (three down the length of the arm and two in the food well). A rat was placed in the maze and allowed to freely navigate and consume the food pellets for $15 \mathrm{~min}$. If a rat consumed all 20 pellets before $15 \mathrm{~min}$, it was removed from the maze and placed in a holding cage, the maze was rebaited with 12 additional pellets, and the rat was placed back in the center of the maze. On the second habituation day, arms were only baited with three pellets each (two in the food wells and one in the center of the arms). Whenever a rat traversed the entire length of an arm and consumed the two food pellets in the well, it was picked up and placed at the entrance of a different arm. This procedure was intended to habituate the animal to repeated handling after consuming food reward. Subsequent habituation sessions were similar to the second day, except that only one food pellet was placed at the end of each arm. This procedure continued daily until the rat had consumed all four food pellets on the maze at least four times in the $15 \mathrm{~min}$ period. Rats required an average of $4.1 \pm 0.2 \mathrm{~d}$ of habituation (range, 3-13 d) to reach this habituation criterion.

After the rat had achieved habituation criterion on the last day of habituation, the turn bias for the rat was determined. The white opaque Plexiglas insert was placed at the entrance of one of the arms, forming a " $\mathrm{T}$ " configuration. A rat was placed in the stem arm and allowed to turn left or right to obtain a food pellet. In one of the choice arms, a blackand-white-striped laminated piece of posterboard $(9 \times 20 \mathrm{~cm})$ serving as a visual cue was placed on the floor. After a rat chose an arm and consumed a food pellet, it was picked up, placed in the stem arm, and allowed to make the next choice. If the rat chose the same arm as the initial choice, it was returned to the stem arm until it chose the other arm and consumed the food pellet. After choosing both arms, the rat was returned to the holding cage, the Plexiglas barrier and visual cue were moved to different arms, and a new trial commenced. Thus, a trial for the turn-bias procedure consisted of entering both choice arms and consuming the food pellets. The turn that a rat made first during the initial choice of a trial was recorded and counted toward its turn bias, and the direction (right or left) that a rat turned four or more times over seven trials was considered its turn bias. After determining the turn bias, a rat's obdurators were removed from the guide cannulas, and two injection cannulas were inserted for $2 \mathrm{~min}$, but no solution was injected at this time. This procedure was performed to habituate the animal to the two infusions they would receive over the next $2 \mathrm{~d}$ of testing. Response (experiments $1 \mathrm{~A}$ and $2 \mathrm{~A}$ ) or visual-cue (experiments $1 \mathrm{~B}$ and $2 \mathrm{~B}$ ) discrimination training commenced on the following day.

\section{Response-visual-cue strategy set-shifting procedure}

The set-shifting task used in the present study has also been described previously (Ragozzino, 2002; Floresco et al., 2006). This task differs from other types of perceptual set-shifting paradigms in that it places a heavier emphasis on response conflict, given that animals are presented the same stimuli during initial discrimination learning and during the set shift, although it also requires the animal to shift attention from one stimulus dimension to another (e.g., turn direction vs visual cues) (Slamecka, 1968). Other perceptual set-shifting studies have used novel complex stimuli during initial discrimination training, reversals, intradimensional and extradimensional shifts (Birrell and Brown, 2000; McAlonan and Brown, 2003). Thus, the set-shift component of the task used in the present study likely engages attentional set-shifting functions mediated by the medial PFC (Ragozzino et al., 1999) but may also include a partial reversal component. We chose this task to directly compare the effects of NAc manipulations to those observed after manipulations of the medial PFC and dorsomedial striatum (Ragozzino et al., 1999, 2002; Floresco et al., 2005, 2006). An added advantage of this task is that it permits a detailed analysis of the different types of errors that rats may commit during the set shift, providing further insight as to whether impairments in behavior are attributable to enhanced perseverative responding or a deficit in acquiring and maintaining a new strategy.

Day 1: response discrimination training. For this discrimination, the animal was required to always turn in the opposite direction of its turn bias (left or right), regardless of the location of the visual cue placed in one of the arms (Fig. 1, top panels). Over the course of training, one of three start arms were used, to discourage animals from using an allocentric spatial strategy to locate the food. On day 1 of training, a rat was started from the arms designated west, south, and east. The location of these arms relative to the spatial cues in the room was varied across animals, so that the maze was placed in one of four possible orientations. For every trial, the visual cue was placed in one of the choice arms so that over every consecutive set of 12 trials it was placed an equal number of times in each choice arm. The order of the start location for each trial, as well as the position of the visual cue, were determined pseudorandomly and taken from a preset sequence that was identical for each animal. On an individual trial, the rat was placed in the stem arm and required to make the appropriate turn to receive a food pellet. Between trials, a rat was placed back in the holding cage on a bench adjacent to the maze. The intertrial interval was $\sim 15 \mathrm{~s}$. A rat continued to receive training trials until it reached a criterion of 10 correct consecutive choices. There was no limit on the number of trials a rat was allotted to reach this criterion. After the rat achieved this acquisition criterion, it received a probe trial; this consisted of starting the rat from the fourth arm (north) that was not used as a start arm during testing. During probe trials, the visual cue was inserted in the arm opposite to the direction that the rat was required to turn. If a rat correctly turned the same direction as was required during training, then response discrimination training was completed. If a rat made an incorrect turn, response training was continued until a rat made an additional five correct choices consecutively, at which time another probe trial was administered. This procedure was continued until a rat made a correct choice on the probe trial. The following measures were 
Response Version (Day 1)
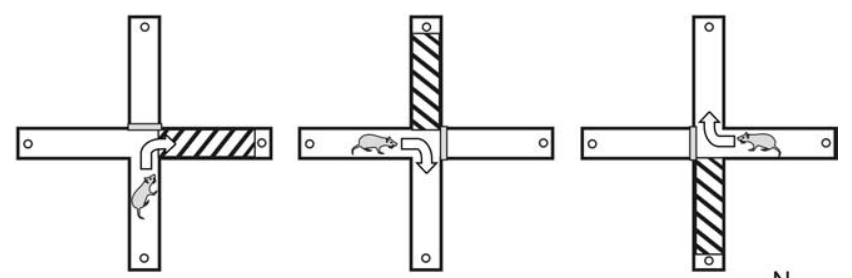

Visual-Cue Version (Day 2)
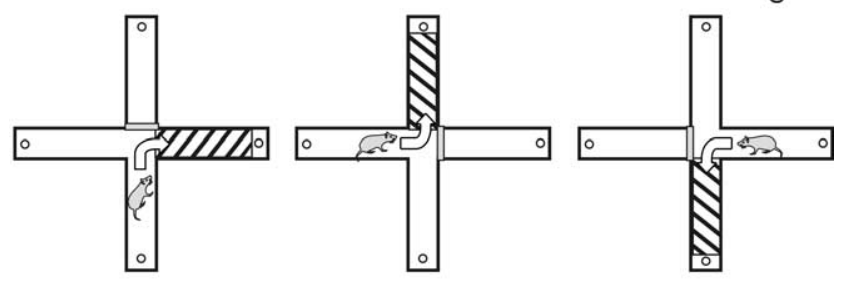

B

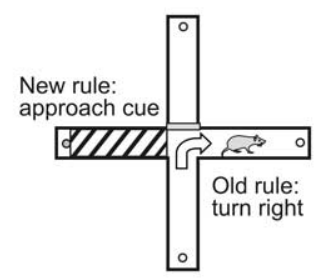

Error Types

Perseverative: early in choice sequence Regressive: after $\leq 50 \%$ perserverative errors

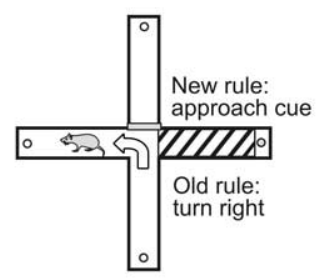

Never-reinforced

Figure 1. Example of the strategy set-shifting task used in experiments $1 A$ and $2 A$. The arrows in the maze represent the correct navigation pattern to receive reinforcement. $A$, During initial response discrimination training on day 1 (top panels), in this example, the rat was started from the south (S), west (W), and east (E) arms and always had to make a $90^{\circ}$ turn to the right to receive food reinforcement. A black-and-white-striped visual cue was placed randomly in one of the choice arms on each trial but did not reliably predict the location of food during response training. During the set shift on day 2 (bottom panels), the rat was required to use a visual-cue discrimination strategy. Here, the rat was started from the same arms but had to always enter the arm with visual cue, which could require either a right or left turn. Thus, the rat must shift from the old strategy and approach the previously irrelevant cue to obtain reinforcement. $\boldsymbol{B}$, Examples of the three types of errors that rats could make during the set shift. See Materials and Methods for details.

taken for each rat and used for data analysis: (1) trials to criterion, defined as the total number of test trials completed before a correct choice on the probe trial was made; and (2) probe trials, defined as the total number of probe trials an animal required to get one correct. The time it took to complete training was also recorded. If a rat did not complete at least 30 trials within the first hour of training, training was discontinued, and the animal's data were excluded from the analysis.

Day 2: shift to visual-cue learning. The day after reaching criterion on the response version, rats were now trained to enter the arm that contained the visual cue, the location of which was pseudorandomly varied in the left and right arms such that it occurred in each arm with equal frequency for every consecutive set of 12 trials (Fig. 1, bottom panels). The training procedure was similar to that used in the response version. The same start arms and criteria to complete the visual-cue version were used as described in the response version. For probe trials, the visual cue was always placed in the arm opposite to that which the rat had been trained to enter during response discrimination training.

Errors were scored as entries into arms that did not contain the visual cue and were further broken down into three error subtypes to determine whether treatments altered the ability to either shift from the previously learned strategy (perseverative errors) or maintain the new strategy after perseveration had ceased (regressive and never-reinforced errors) (Fig.
1B). A "perseverative" error was scored when a rat continued to make the same egocentric response as required on the response version on those trials that required the rat to turn in the opposite direction to enter the arm containing the visual cue (Fig. $1 B$, left). Six of every 12 consecutive trials required the rat to respond in this manner (i.e., enter the arm opposite of the previously learned turn direction). As described in previous studies (Ragozzino et al., 1999; Dias and Aggleton 2000; Ragozzino, 2002; Floresco et al., 2006), these trials were separated into consecutive blocks of four trials each. Perseverative errors were scored when a rat entered the incorrect arm on three or more trials per block of four trials. Once a rat made less than three perseverative errors in a block for the first time, all subsequent errors were no longer counted as perseverative errors because, at this point, the rat was choosing an alternative strategy at least half of the time. Instead, these errors were now scored as "regressive" errors. The third type of error, termed "never-reinforced" errors, was scored when a rat entered the incorrect arm on trials in which the visual cue was placed in the same arm that the rat had been trained to enter on the previous day. For example, during training on day 1 , a rat might be required to turn right. During the shift on day 2 , a rat must enter the arm with the visual cue, and for half of the trials, the cue was in the right arm. In this situation, a never-reinforced error was scored when a rat entered the left arm (i.e., a choice that was not reinforced on either day 1 or day 2) (Fig. $1 B$, right). Both regressive and never-reinforced errors are used as an index of the animals' ability to acquire and maintain a new strategy.

\section{Visual-cue-response strategy set-shifting procedure}

For these experiments, rats were initially trained on the visual-cue version of the task on day 1 , followed by testing on the response version on day 2. All other aspects of the testing procedure were identical to those described above. On the shift to the response version, the same measures were assessed as those for experiment 1 , in which rats were required to shift from a response to a visual-cue strategy. However, perseverative and regressive errors were analyzed from the trials in which a rat was required to turn in the arm opposite to that of the visual cue.

\section{Microinfusion procedure and experimental design}

Inactivation of the NAc core or shell was achieved by infusion of a drug solution containing the $\mathrm{GABA}_{\mathrm{B}}$ agonist baclofen (Sigma-Aldrich Canada, Oakville, Ontario, Canada) and the $\mathrm{GABA}_{\mathrm{A}}$ agonist muscimol (Sigma-Aldrich). GABA ${ }_{A}$ and $\mathrm{GABA}_{\mathrm{B}}$ receptors are expressed in both the core and shell regions of the NAc to a similar degree, although there are regional differences in the distribution of $\alpha$-type subunits of the $\mathrm{GABA}_{\mathrm{A}}$ receptor (Kaufmann et al., 2003; Amantea et al., 2004). Given the relatively high concentration of both drugs used in the present study, it would be expected that infusion of these compounds into either the NAc core or the NAc shell would cause an equivalent suppression of neural activity in both regions.

Both drugs were dissolved in physiological saline and protected from light. Each drug was mixed separately at a concentration of $500 \mathrm{ng} / \mu \mathrm{l}$ and combined in equal volumes, so that the final concentration of each compound in solution was $250 \mathrm{ng} / \mu \mathrm{l}$. For intracranial microinfusions, we used a volume of $0.3 \mu \mathrm{l}$, so that the final dose of both baclofen and muscimol was $75 \mathrm{ng}$ per side. Previous studies have shown that infusion of these compounds at this volume induces dissociable effects on behavior when administered into the NAc core or shell (McFarland and Kalivas, 2001).

Infusions of GABA agonists or saline were administered bilaterally into either the NAc core (experiments $1 \mathrm{~A}$ and $2 \mathrm{~A}$ ) or the NAc shell (experiments $1 \mathrm{~B}$ and $2 \mathrm{~B}$ ) via 30 gauge injection cannulas that protruded $0.8 \mathrm{~mm}$ past the end of the guide cannulas, at a rate of $0.3 \mu \mathrm{l} / 46 \mathrm{~s}$ by a microsyringe pump (model 341; Sage Instruments, Cambridge, MA). Injection cannulas were left in place for an additional $1 \mathrm{~min}$ to allow for diffusion. Each rat remained in its home cage for an additional $10 \mathrm{~min}$ period before behavioral testing.

Each experimental series used three groups of rats. The control group received infusions of saline into the NAc core or shell before initial discrimination training on day 1 and during the set shift on day 2 . A second group received saline infusions on day 1 and inactivation of the NAc core 
or shell before the set shift on day 2. A third group of rats received inactivation of the NAc core or shell on day 1 and a saline infusion before the set shift on day 2, to assess the effects of inactivation of the NAc core or shell on the initial learning of a simple discrimination.

\section{Histology}

After completion of behavioral testing, the rats were killed in a carbon dioxide chamber. Brains were removed and fixed in a $4 \%$ formalin solution. The brains were frozen and sliced in 50 $\mu \mathrm{m}$ sections before being mounted and stained with cresyl violet. Placements were verified with reference to the neuroanatomical atlas of Paxinos and Watson (1998). Rats with cannula placements that were too ventral were excluded from the data analysis. The locations of infusions for all rats with acceptable placements in experiments 1 and 2 are shown in Figure 2.

\section{Data analysis}

The number of trials to reach criterion on day 1 of training for each experiment were analyzed separately using a one-way ANOVA to assess the effects of inactivation of the NAc core or shell on the initial learning of a response or visual-cue discrimination. Trials to criterion data obtained from day 2 (set shift) for each experiment were analyzed using separate twoway between/within-subjects ANOVA, with treatment as the between-subjects factor and choice type (correct or error) as the withinsubjects factor. The number of errors committed during the set shift was analyzed separately using a one-way betweensubjects ANOVA, with treatment as the between-subjects factor. When a significant main effect of treatment was observed, multiple comparisons were conducted using Dunnett's test. A priori, we were specifically interested in how inactivation of NAc subregions would alter each type of error, to obtain a better understanding of the specific processes that may be altered during set shifting. Thus, when a main effect of treatment was observed, we performed separate analyses on the number of each type of error. For measures of response latency, the total number of trials required to reach criterion was divided by the total time required to reach criterion (trials per minute). These data were analyzed using separate one-way ANOVAs.

\section{Results}

Experiment 1A: effects of inactivation of the NAc core on shifting from a response to a visual-cue strategy

\section{Day 1: response discrimination training}

Analysis of the number of trials required to achieve criterion performance by rats receiving infusions of saline or baclofen/ muscimol into the NAc core revealed no significant main effect of treatment $\left(F_{(2,17)}=0.90\right.$; NS). Rats receiving inactivation of the NAc core on day $1(n=6)$ were not impaired in learning a simple response discrimination relative to control rats $(n=7)$ or rats that would receive NAc core inactivations on day $2(n=7)$ (Fig. $3 A)$. However, inactivation of the NAc core did decrease the number of trials completed per minute. Saline-treated rats completed $1.6 \pm 0.1$ trials per minute, whereas rats receiving inactivation of the NAc core were substantially slower, completing $0.7 \pm 0.1$ trials per minute $\left(F_{(2,17)}=23.12 ; p<0.001\right.$ and Dunnett's test, $p<0.001)$. There were no differences between groups in the number of probe trials required to complete training on day $1\left(F_{(2,17)}=0.16\right.$; NS). Thus, inactivation of the NAc core does not disrupt learning a simple egocentric response strategy.

\section{Day 2: shift to visual-cue discrimination}

Analysis of the number of trials to reach criterion on day 2 revealed a significant main effect of treatment $\left(F_{(2,17)}=8.68 ; p<\right.$ $0.005)$. There was also a significant effect of choice type $\left(F_{(1,17)}=\right.$ 138.28; $p<0.001)$, indicating that all rats made more correct than incorrect choices but no significant treatment-by-choicetype interaction $\left(F_{(2,17)}=2.17 ; \mathrm{NS}\right)$. Dunnett's tests revealed that rats receiving inactivation of the NAc core before the set shift on day $2(n=7)$ required significantly more trials to reach criterion compared with control rats $(n=7 ; p<0.01)$, whereas rats receiving inactivation of the NAc core on day 1 and saline on day 2 $(n=6)$ did not differ from controls on this measure (Fig. $3 B)$.

A separate analysis of the errors committed during the set shift revealed a significant main effect of treatment $\left(F_{(2,17)}=5.29 ; p<\right.$ $0.05)$. Multiple comparisons revealed that rats receiving infusions of baclofen/muscimol into the NAc core before the set shift made significantly more errors than those receiving saline treatments $(p<0.05)$, consistent with the trials to criterion data. Subsequent analyses on each type of error revealed that NAc core inactivation did not alter the number of perseverative errors relative to control treatments $\left(t_{(12)}=0.37\right.$; NS) but did significantly enhance the number of regressive and never-reinforced errors $\left(t_{(12)}=2.33 ; p<0.05\right.$ ) (Fig. $3 C$ ). A separate one-way ANOVA conducted on the latency data showed no significant difference between treatment groups on the average number of trials completed per minute $\left(F_{(2,17)}=2.64\right.$; NS), or the number of probe trials required to complete training on day $2\left(F_{(2,17)}=0.82 ; \mathrm{NS}\right)$. Thus, inactivation of the NAc core impairs shifting from a response to a visual-cue-based discrimination strategy, but this effect is not attributable to an increase in perseverative responding. Rather, this effect is caused by an increase in both regressive and 

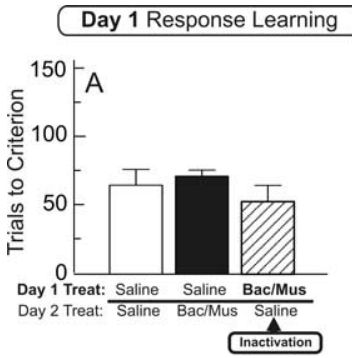

Day 1 Visual Cue Learning

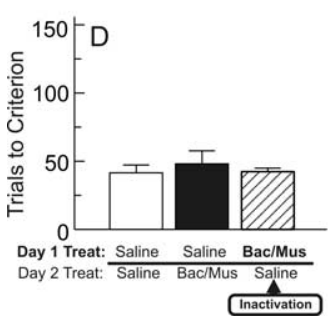

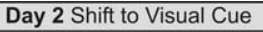
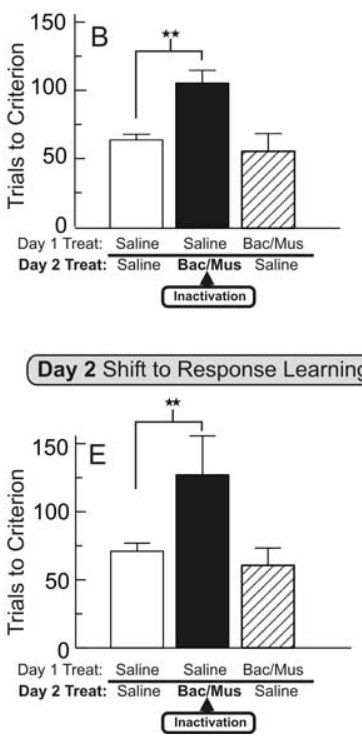

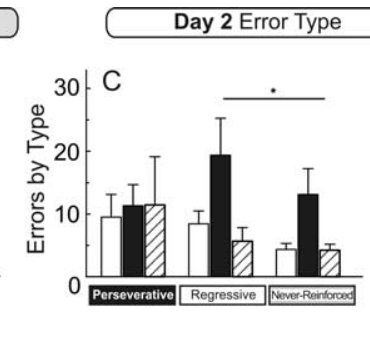

\section{g)}
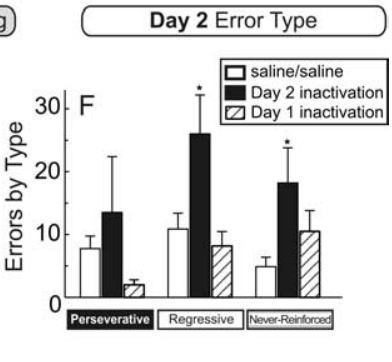

Figure 3. Experiment 1: inactivation of the NAc core disrupts shifting from a response to a visual-cue-based strategy (experiment $1 \mathrm{~A} ; \boldsymbol{A}-\boldsymbol{C}$ ) and a visual-cue to a response strategy (experiment $1 \mathrm{~B} ; \boldsymbol{D}-\boldsymbol{F}$ ). Data are expressed as means \pm SEM. $\boldsymbol{A}$, Trials to criterion on acquisition of a response discrimination on day 1 by rats receiving infusions of saline (open and filled bars) or baclofen and muscimol into the NAc core (hatched bar). Inactivation of the core did not impair response learning. $\boldsymbol{B}$, Trials to criterion on the shift to visual-cue discrimination strategy on day 2 after infusions of either saline (open and hatched bars) or baclofen and muscimol into the NAc core (filled bar). ${ }^{\star \star} p<0.01$, significantly different from the saline control group. $\boldsymbol{C}$, Analysis of the type of errors committed in experiment $1 A$ during the set shift on day 2. Inactivation of the NAc core before the set shift (filled bars) did not alter the number of perseverative errors (left) but increased the number of regressive (middle) and never-reinforced (right) errors. ${ }^{\star} p<0.05$, significantly different from combined errors made by saline-treated rats (open bars). Inactivation of the core before initial discrimination learning (hatched bars) did not alter the number of errors during the set shift. $\boldsymbol{D}$, Trials to criterion on acquisition of the visual-cue discrimination on day 1 by rats receiving either infusions of saline (open and filled bars) or baclofen and muscimol into the NAc core (hatched bar). Inactivation of the core did not impair visual-cue-based learning. $\boldsymbol{E}$, Trials to criterion on the shift to the response discrimination on day 2 after infusions of either saline (open and hatched bars) or baclofen and muscimol into the NAc core (filled bar). ${ }^{\star \star} p<0.01$, significantly different from the saline control group. $\boldsymbol{F}$, Again, NAc core inactivation did not effect perseveration (left) but increased the number of regressive (middle) and never-reinforced (right) errors during the shift on day 2 ( ${ }^{\star} p<0.05$, significantly different from the same type of errors made by saline-treated rats). Treat, Treatment; Bac/Mus, baclofen/muscimol.

never-reinforced errors, indicative of an impairment of acquiring and maintaining a new strategy.

\section{Experiment 1B: effects of inactivation of the NAc core on shifting from a visual cue to a response strategy \\ Day 1: visual-cue discrimination training}

Rats receiving inactivation of the NAc core on Day $1(n=6)$ took an equivalent number of trials to learn a visual-cue discrimination relative to control rats $(n=8)$ or rats that would receive NAc core inactivations on day $2\left(n=6 ; F_{(2,17)}=0.29\right.$; NS) (Fig. $\left.3 D\right)$. Moreover, in this instance, NAc core inactivation did not affect the number of trials completed per minute $(1.1 \pm 0.2)$ relative to saline-treated rats $\left(1.2 \pm 0.1 ; F_{(2,17)}=0.77 ; \mathrm{NS}\right)$. There were also no differences between groups in the number of probe trials required to complete training on day $1\left(F_{(2,17)}=0.80\right.$; NS). Thus, inactivation of the NAc core does not disrupt initial visual-cue discrimination learning.

\section{Day 2: shift to response discrimination}

Analysis of the number of trials to reach criterion on day 2 revealed a significant main effect of treatment $\left(F_{(2,17)}=4.26 ; p<\right.$ $0.05)$. There was also a significant effect of choice type $\left(F_{(1,17)}=\right.$ 18.04; $p<0.001)$ but no significant treatment-by-choice-type interaction $\left(F_{(2,17)}=0.69\right.$; NS). Multiple comparisons revealed that rats receiving inactivation of the NAc core before the set shift on day $2(n=6)$ required significantly more trials to reach criterion compared with control rats $(n=8 ; p<0.01)$, whereas rats receiving inactivation of the NAc core on day 1 and saline on day $2(n=$ 6) did not differ from controls on this measure (Fig. 3E).

Analysis of the errors committed during the set shift revealed a significant main effect of treatment $\left(F_{(2,17)}=6.01 ; p<\right.$ $0.05)$. Inactivation of the NAc core induced a significant increase in the total number of errors relative to control rats $(p<0.05)$. Consistent with the findings of experiment $1 \mathrm{~A}$, these treatments did not increase the number of perseverative errors relative to control treatment $\left(_{(12)}=\right.$ 0.73 ; NS) but did significantly increase the number of regressive $\left(t_{(12)}=2.51 ; p<\right.$ $0.05)$ and never-reinforced errors $\left(t_{(12)}=\right.$ $2.61 ; p<0.05)$ (Fig. $3 F$ ). NAc core inactivation did not increase response latencies $\left(F_{(2,17)}=0.77\right.$; NS), nor did it alter the number of probe trials required to complete training on day $2\left(F_{(2,17)}=0.69 ; \mathrm{NS}\right)$. Thus, inactivation of the NAc core impairs shifting from a visual-cue- to a responsebased discrimination strategy. As observed in experiment $1 \mathrm{~A}$, this manipulation increased regressive and neverreinforced errors but did not enhance perseverative responding. These impairments cannot be attributed to a disruption of motoric or motivation processes or deficits in response or visual-cue discrimination learning, because the same treatments on day 1 did not impair learning. Together, the results of experiments $1 \mathrm{~A}$ and $1 \mathrm{~B}$ suggest that the NAc core mediates learning and maintenance of a novel discrimination only when animals are required to disregard a previously relevant strategy and attend to previously irrelevant stimuli to obtain a goal.

\section{Experiment 2A: effects of inactivation of the NAc shell on} shifting from a response to a visual-cue strategy

Day 1: response discrimination training

Analysis of the trials to criterion data by rats receiving infusions of either saline or baclofen/muscimol into the NAc shell revealed no significant main effect of treatment $\left(F_{(2,16)}=1.16\right.$; NS). Rats receiving inactivation of the NAc shell on day $1(n=7)$ learned a response discrimination as readily as control rats $(n=6)$ or rats that would receive inactivations on day $2(n=6)$ (Fig. $4 A$ ). Although inactivation of the NAc shell increased the number of trials completed per minute (mean, $1.8 \pm 0.2$ ), this did not differ significantly from rats treated with saline (mean, $1.3 \pm 0.2$; $F_{(2,16)}=2.24$; NS). There were also no differences between groups on the number of probe trials required to complete training on day $1\left(F_{(2,16)}=0.53\right.$; NS). Thus, like the NAc core, the NAc shell does not play a role in learning a simple egocentric response strategy.

\section{Day 2: shift to visual-cue discrimination}

Analysis of the number of trials to reach criterion during the set shift on day 2 revealed a significant main effect of treatment 
$\left(F_{(2,16)}=5.63 ; p<0.05\right)$. There was also a significant effect of choice type $\left(F_{(2,16)}=\right.$ $144.14 ; p<0.001)$, but the treatment-bychoice-type interaction was not significant $\left(F_{(2,17)}=1.10\right.$; NS). Multiple comparisons on the main effect of treatment revealed that rats receiving inactivation of the NAc shell before the set shift on day 2 $(n=6)$ were not impaired relative to control rats $(n=6)$. Surprisingly, however, rats that had received an inactivation of the NAc shell on day 1 and saline on day 2 $(n=7)$ displayed better performance during the set shift relative to control rats, requiring fewer trials to reach criterion $(p<$ 0.001 ) (Fig. $4 B$ ). In fact, rats in this group actually required significantly fewer trials to achieve criterion on the visual-cue discrimination during the set shift $(24.7 \pm 4)$ than rats that received saline infusions before initial visual-cue discrimination training on day 1 in experiment $2 \mathrm{~B}$ $\left(43.7 \pm 5 ; t_{(18)}=2.59 ; p<0.05\right)$.

Analysis of the errors committed during the set shift revealed a significant main effect of treatment $\left(F_{(2,16)}=7.52 ; p<\right.$ $0.005)$. Consistent with the trials to criterion data, rats receiving infusions of baclofen/muscimol into the NAc shell on day 1 made significantly fewer errors than control rats $(p<0.05)$. On the other hand, rats receiving infusions of baclofen/ muscimol into the NAc shell before the set shift made a comparable number of errors compared with control rats. Analyses of each type of error revealed that the improved performance by rats that had received NAc shell inactivations on day 1 was attributable to a decrease in the number of both perseverative $\left(t_{(11)}=2.94 ; p<0.05\right)$ and regressive $\left(t_{(11)}=2.43 ; p<0.05\right)$ errors relative to control rats (Fig. $4 C$ ). There were no significant differences between treatment groups on either the average number of trials completed per minute $\left(F_{(2,16)}=2.40\right.$; NS) or the number of probe trials required to complete training on day $2\left(F_{(2,16)}=0.58\right.$; NS).

\section{Experiment 2B: effects of inactivation of the NAc shell on shifting from a visual-cue to a response strategy}

Day 1: visual-cue discrimination training

Similar to the results of experiment $2 \mathrm{~A}$, infusions of baclofen/ muscimol into the NAc shell did not affect the number of trials required to achieve criterion performance on the visual-cue discrimination relative to saline-treated rats $\left(F_{(2,16)}=0.48\right.$; NS) (Fig. $4 D$ ). There was also no difference between groups on the number of trials completed per minute or the number of probe trials required to complete training on day 1 (all $F<1$; NS).

\section{Day 2: shift to response discrimination}

Analysis of the number of trials to criterion during the set shift on day 2 revealed a significant main effect of treatment $\left(F_{(2,16)}=\right.$ $5.63 ; p=0.05)$. There was also a significant effect of choice type $\left(F_{(1,16)}=85.13 ; p<0.001\right)$ but no significant treatment-bychoice-type interaction $\left(F_{(2,16)}=0.43\right.$, n.s. $)$. Rats receiving inac-
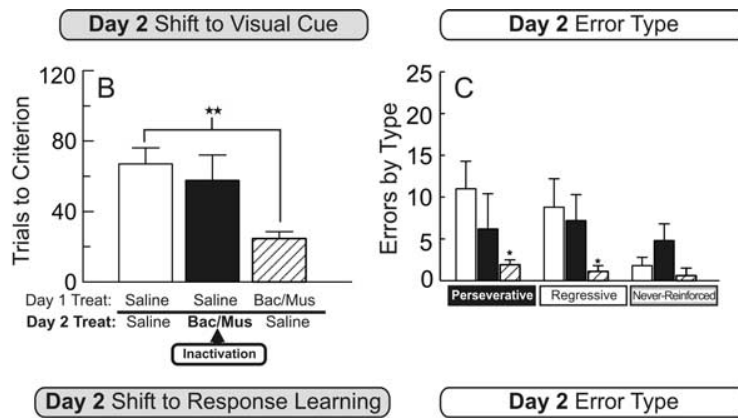

Day 2 Error Type
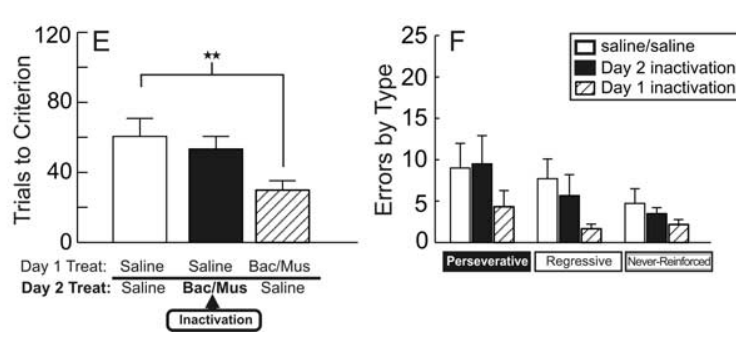

Figure 4. Experiment 2: effects of inactivation of the NAc shell on shifting from a response to a visual-cue-based strategy (experiment $2 \mathrm{~A} ; \boldsymbol{A}-\boldsymbol{C}$ ) and a visual-cue to a response strategy (experiment $2 \mathrm{~B} ; \boldsymbol{D}-\boldsymbol{F}$ ). Data are expressed as means \pm SEM. $\boldsymbol{A}$, Trials 位

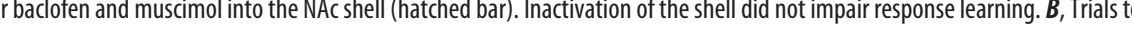
receiving inactivation of the shell on day 1 and saline infusions on day 2 (hatched bar) displayed improved performance during the set shift. ${ }^{\star \star} p<0.01$, significantly different from the saline control group. C, Analysis of the type of errors committed in experi列

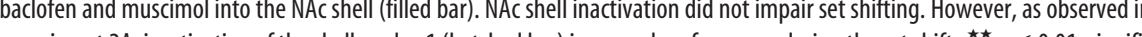
shift on day 2 by each group of rats. Treat, Treatment; Bac/Mus, baclofen/muscimol.

tivation of the NAc shell before the set shift $(n=7)$ were not impaired relative to control rats $(n=6)$. Furthermore, replicating the effects observed in experiment $2 \mathrm{~A}$, rats receiving inactivation of the NAc shell on day 1 and saline on day $2(n=6)$ displayed better performance during the set shift compared with control rats, requiring fewer trials to reach criterion $(p<0.005)$ (Fig. $4 E$ ). Rats in this group also required significantly fewer trials to learn the response discrimination during the set shift (29.8 \pm 5 ) than rats that received saline infusions before initial response discrimination training on day 1 in experiment $2 \mathrm{~A}\left(60.3 \pm 6 ; t_{(16)}=\right.$ $3.12 ; p<0.01)$. These treatments also resulted in fewer perseverative and regressive errors during the set shift, but the difference in the total number of errors between groups only approached statistical significance $\left(F_{(2,16)}=2.99 ; 0.05<p<0.10\right)$ (Fig. $4 F$ ). There were no significant differences between groups on either the average number of trials completed per minute or the number of probe trials required to complete training on day 2 (all $F<1.9$; NS). Thus, unlike the NAc core, the NAc shell does not mediate shifting from a response to a visual-cue-based discrimination strategy or vice versa. However, inactivation of the NAc shell during initial discrimination training improves performance during the set shift relative to control rats.

One possible explanation for the improvements reported here may be that inactivation of the NAc shell may have disrupted the 
consolidation of the initial strategy (Setlow and McGaugh, 1998; Hernandez et al., 2002; Dalley et al., 2005). The observation that NAc shell inactivation reduced perseveration during the set shift indicates that rats were less likely to respond in a manner consistent with the initial discrimination rule, which may be interpreted as impaired consolidation of the previously acquired strategy. However, it is important to note that inactivation of the NAc shell did not (1) disrupt initial discrimination learning or (2) impair or improve learning during the set shift. Moreover, previous studies implicating the NAc in consolidation have used post-training manipulations, whereas we inactivated the NAc shell before initial discrimination training. Also, rats in these groups learned faster during the set shift than controls during initial discrimination training on day 1 , indicating that rats receiving NAc shell inactivation experienced some benefit from their initial training. Thus, although we cannot rule out that the improvements in set shifting may be attributable to impaired consolidation processes, this would suggest that the NAc shell is involved selectively only in the consolidation, but not acquisition or retrieval, of response or visual-cue strategies. Furthermore, the fact that discrete microinfusions of baclofen and muscimol into adjacent subregions of the NAc resulted in dissociable effects on behavior indicates that the inactivation procedure used here was sufficient to induce selective inactivation of the core and shell.

\section{Discussion}

Here, we report dissociable effects induced by inactivation of the NAc core or shell on set shifting. Inactivation of either subregion does not impair learning of a response or visual-cue discrimination. However, inactivation of the core disrupted shifting from one strategy to another, whereas similar manipulations of the shell did not impair set shifting. Most surprising were the findings that inactivation of the NAc shell before initial discrimination learning led to improved performance during the set shift.

\section{The NAc core as part of a neural circuit that mediates behavioral flexibility}

Manipulations of the medial PFC impair set shifting using a protocol similar to that used in the present study, selectively increasing perseverative errors (Ragozzino et al., 1999; Ragozzino, 2002; Floresco et al., 2006), suggesting that the PFC facilitates extinction of a previously relevant strategy. In contrast, inactivation of the NAc core increased both regressive and never-reinforced errors. Although both perseverative and regressive errors entail a choice consistent with the previously acquired, but now incorrect, strategy, a key difference is when these errors occur in the choice sequence. Regressive errors occur later in the choice sequence, when rats use the previous strategy on fewer than $50 \%$ of trials in which the correct response is opposite to that required using the initial rule. The fact that core inactivation increased regressive but not perseverative errors implies that this subregion does not mediate the suppression of a previously acquired response. Moreover, similar inactivation on day 1 did not disrupt the initial acquisition of either discrimination. Thus, the NAc core seems to play a selective role in mediating flexibility by facilitating the generation and maintenance of novel behavioral responses that conflict with previously acquired strategies. Interestingly, inactivation of the dorsomedial striatum causes a similar increase in regressive errors (Ragozzino et al., 2002). Together, it is apparent that both dorsal and ventral striatal regions act cooperatively to facilitate the generation and maintenance of novel behavioral strategies in which an organism must choose between conflicting responses. The interaction between these two regions may be subserved by descending projections from the NAc core that can influence the dorsal striatum via spiraling projections to nigrostriatal dopamine neurons (Haber et al., 2000). Thus, the NAc core may function as part of a larger striatal complex, mediating the selection of relevant actions among competing motor responses (Mink and Thach, 1993).

As opposed to perseverative/regressive errors, neverreinforced errors entail a choice that was incorrect during both initial discrimination training and during the shift. Rats quickly learn that the previously correct strategy is no longer appropriate and engage alternative strategies until the appropriate solution is resolved. Never-reinforced errors may be interpreted as an attempt to use alternative strategies, typically a reversal of the previously acquired rule (e.g., always turn right instead of left). Intact rats make relatively few errors of this type and learn that this strategy does not lead to reward reliably. In contrast, core inactivations increased never-reinforced errors, indicating that the core also mediates the elimination of inappropriate response options, enabling the reorganization of behavior to obtain reward in an optimal manner.

The set-shift component of this task also entailed a partial reversal of the previously rewarded response. Thus, it is possible that impairments observed here may also be attributable to a disruption in reversal learning mediated by the orbital PFC. In this regard, the role of the orbital PFC in this type of strategy set shifting is currently unknown. However, previous studies have shown that NAc lesions do not impair motor, odor, or visual reversals (Stern and Passingham, 1995; Schoenbaum and Setlow, 2003) and cause variable effects on spatial reversals (Annett et al., 1989; Burk and Mair, 2001). However, NAc lesions cause nonperseverative impairments when rats must reverse from a match to a nonmatching strategy, indicating an impairment of "higherorder response organization" (Reading and Dunnett, 1991). Thus, it is likely that the deficits induced by core inactivations were attributable primarily to a disruption in learning to use a previously incorrect discrimination strategy during the set shift, rather than an impairment of reversal learning per se.

Compared with the present data, impairments in set shifting induced by inactivation of the PFC or dorsomedial striatum are not associated with an increase in the number of neverreinforced errors (Ragozzino et al., 1999, 2002). The fact that inactivation of each of these brain regions induces distinct types of deficits suggests that the NAc core is part of a distributed corticostriatal network that interacts cooperatively to facilitate strategy shifting. However, each of these regions makes dissociable contributions to the cognitive processes that enable an animal to alter behavior. The PFC plays an essential role early in set shifting, suppressing the use of a previously correct strategy (Dias et al., 1997; Ragozzino et al., 1999). Once the animal attends to alternative stimuli that may be predictive of reward, the NAc core enables new learning by facilitating the elimination of inappropriate strategies. Subsequently, when the appropriate strategy has been ascertained, maintenance of the novel strategy is likely mediated by parallel corticostriatal circuits that include the dorsomedial striatum and NAc core (Ragozzino et al., 2002; Reynolds and Zahm, 2005).

\section{Improvements in strategy set shifting induced by inactivation of the NAc shell}

In contrast to the effects of NAc core inactivation, similar manipulations of the NAc shell did not impair set shifting. However, inactivation of the shell before initial discrimination training on day 1 improved performance in these animals during the set shift. 
Although an unexpected finding, there have been other reports that lesions of the NAc shell can improve associative learning. Studies of latent inhibition have shown that pre-exposure to a "to be" conditioned stimulus in the absence of reinforcement subsequently impedes associative learning (Weiner, 1990; Lubow, 1997). Thus, "normal" latent inhibition is expressed as a retardation of learning, presumably because the animal learns to ignore irrelevant stimuli that predict no significant consequences (Weiner and Feldon, 1997). However, lesions to the NAc shell (but not core) abolish this effect; animals learn the association much more rapidly (Tai et al., 1995; Weiner et al., 1996; JongenRelo et al., 2002), suggesting that the NAc shell mediates learning to ignore stimuli that do not reliably predict reinforcement (i.e., learned irrelevance) (Owen et al., 1993; Bennett et al., 1995; Maes et al., 2004). A similar phenomenon may underlie the findings observed here. During initial discrimination training, a rat associates particular stimuli with availability of reward, whereas, at the same time, learning to ignore irrelevant stimuli. Normally, this learned irrelevance would impede initial learning during the set shift, in which rats must now approach the previously irrelevant stimulus. Yet, inactivation of the NAc shell on day 1 improved learning during the set shift relative to controls, similar to shell-lesioned animals subjected to tests of latent inhibition (Tai et al., 1995; Jongen-Relo et al., 2002). Thus, it is plausible that improvements in set shifting reported here may be attributable in part to a failure to encode the irrelevance of stimuli during initial learning, which in turn would enhance learning about the relevance of these stimuli during the set shift.

The present findings suggest that decreased activity in the NAc shell during initial discrimination learning can improve learning of an alternate discrimination during set shifting. Thus, it is plausible that increased activation of the NAc shell may exert the opposite effect, augmenting initial learning that in turn may impede the ability to shift off this strategy during a set shift. Indeed, unilateral inactivation of the PFC combined with contralateral dopamine $\mathrm{D}_{2}$ receptor stimulation in the NAc impairs shifting from a visual-cue to a response strategy (Goto and Grace, 2005). It is interesting to note that schizophrenia, which has long been attributed to an increase in mesoaccumbens dopamine activity, is also associated with impairments in set shifting (Elliot et al., 1995; Pantelis et al., 1999). Although these deficits have been attributed to impaired PFC function, in light of the present data, it is reasonable to propose that the impairments in cognitive flexibility observed in schizophrenia may also be due in part to enhanced activity of the NAc shell.

\section{Conclusion}

Recent studies exploring the neural substrates of behavioral flexibility provide important insight to the contributions that distinct components of corticostriatal circuits make to this type of executive functioning. Cools et al. (2004) have proposed that the $\mathrm{PFC}$ and ventral striatum function in a complementary manner during attentional switching tasks, with the striatum mediating specific stimulus-response associations, whereas the PFC exerts "a more general biasing role in the context of changing environments." Similarly, performance of the Wisconsin Card Sorting Task is associated with distinct patterns of activation in the medial and dorsolateral PFC, the striatum, and the thalamus that are associated with separate stages of the task (Monchi et al., 2001). These authors concluded that "the basal ganglia are particularly important in determining attentional set and guiding action in response to behavioral rules, in conjunction with the $\mathrm{PFC}$ " ( $\mathrm{p}$. 7739). In keeping with this notion, the present data highlight the dissociable contributions of the NAc core and shell to distributed neural circuits that mediate behavioral flexibility, with the NAc shell mediating learning about irrelevant stimuli, and the core facilitating the acquisition and maintenance of novel behavioral strategies.

\section{References}

Amantea D, Tessari M, Bowery NG (2004) Reduced G-protein coupling to the $\mathrm{GABA}_{\mathrm{B}}$ receptor in the nucleus accumbens and the medial prefrontal cortex of the rat after chronic treatment with nicotine. Neurosci Lett 355:161-164.

Annett LE, McGregor A, Robbins TW (1989) The effects of ibotenic acid lesions of the nucleus accumbens on spatial learning and extinction in the rat. Behav Brain Res 31:231-242.

Bennett CH, Maldonado A, Mackintosh NJ (1995) Learned irrelevance is not the sum of exposure to CS and US. Q J Exp Psychol B 48:117-128.

Birrell JM, Brown VJ (2000) Medial frontal cortex mediates perceptual attentional set shifting in the rat. J Neurosci 20:4320-4324.

Brog JS, Salyapongse A, Deutch A, Zahm DS (1993) The pattern of afferent innervation of the core and shell in the "accumbens" part of the ventral striatum: immunohistochemical detection of retrogradely transported Fluoro-gold. J Comp Neurol 338:255-278.

Brown VJ, Bowman EM (2002) Rodent models of prefrontal cortical function. Trends Neurosci 25:340-343.

Burk JA, Mair RG (2001) Effects of dorsal and ventral striatal lesions on delayed matching trained with retractable levers. Behav Brain Res 122:67-78.

Chudasama Y, Bussey TJ, Muir JL (2001) Effects of selective thalamic and prelimbic cortex lesions on two types of visual discrimination and reversal learning. Eur J Neurosci 14:1009-1020.

Cools R, Clark L, Robbins TW (2004) Differential responses in human striatum and prefrontal cortex to changes in object and rule relevance. J Neurosci 24:1129-1135.

Dalley JW, Laane K, Theobald DE, Armstrong HC, Corlett PR, Chudasama Y, Robbins TW (2005) Time-limited modulation of appetitive Pavlovian memory by D1 and NMDA receptors in the nucleus accumbens. Proc Natl Acad Sci USA, 102:6189-6794.

Dias R, Aggleton JP (2000) Effects of selective excitotoxic prefrontal lesions on acquisition of nonmatching- and matching-to-place in the T-maze in the rat: differential involvement of the prelimbic-infralimbic and anterior cingulate cortices in providing behavioral flexibility. Eur J Neurosci 12:4457-4466

Dias R, Robbins TW, Roberts AC (1997) Dissociable forms of inhibitory control within prefrontal cortex with an analog of the Wisconsin Card Sort Test: restriction to novel situations and independence from "online” processing. J Neurosci 17:9285-9297.

Dunnett SB (1990) Role of prefrontal cortex and striatal output systems in short-term memory deficits associated with ageing, basal forebrain lesions, and cholinergic-rich grafts. Can J Psychol 44:210-232.

Elliott R, McKenna PJ, Robbins TW, Sahakian BJ (1995) Neuropsychological evidence for frontostriatal dysfunction in schizophrenia. Psychol Med, 25:619-630.

Floresco SB, Blaha CD, Yang CR, Phillips AG (2001) Modulation of hippocampal and amygdalar-evoked activity of nucleus accumbens neurons by dopamine: cellular mechanisms of input selection. J Neurosci 21:2851-2860.

Floresco SB, Magyar O, Ghods-Sharifi S, Vexelman C, Tse MT (2006) Multiple dopamine receptor subtypes in the medial prefrontal cortex of the rat regulate set-shifting. Neuropsychopharmacology 31:297-309.

Goto Y, Grace AA (2005) Dopaminergic modulation of limbic and cortical drive of nucleus accumbens in goal-directed behavior. Nat Neurosci 8:805-812.

Haber SN, Fudge JL, McFarland NR (2000) Striatonigrostriatal pathways in primates form an ascending spiral from the shell to the dorsolateral striatum. J Neurosci 20:2369-2382.

Hernandez PJ, Sadeghian K, Kelley AE (2002) Early consolidation of instrumental learning requires protein synthesis in the nucleus accumbens. Nat Neurosci 5:1327-1331.

Jongen-Relo AL, Kaufmann S, Feldon J (2002) A differential involvement of the shell and core subterritories of the nucleus accumbens of rats in attentional processes. Neuroscience 111:95-109.

Kaufmann WA, Humpel C, Alheid GF, Marksteiner J (2003) Compartmen- 
tation of alpha 1 and alpha $2 \mathrm{GABA}_{\mathrm{A}}$ receptor subunits within rat extended amygdala: implications for benzodiazepine action. Brain Res 964:91-99.

Lawrence AD, Sahakian BJ, Rogers RD, Hodge JR, Robbins TW (1999) Discrimination, reversal, and shift learning in Huntington's disease: mechanisms of impaired response selection. Neuropsychologia 37:1359-1374.

Lubow RE (1997) Latent inhibition as a measure of learned inattention: some problems and solutions. Behav Brain Res 88:75-83.

Maes JH, Damen MD, Eling PA (2004) More learned irrelevance than perseveration errors in rule shifting in healthy subjects. Brain Cogn 54:201-211.

McAlonan K, Brown VJ (2003) Orbital prefrontal cortex mediates reversal learning and not attentional set shifting in the rat. Behav Neurosci 146:97-103.

McFarland K, Kalivas PW (2001) The circuitry mediating cocaine-induced reinstatement of drug-seeking behavior. J Neurosci 21:8655-8663.

Miller EK (2000) The prefrontal cortex and cognitive control. Nat Rev Neurosci 1:59-65.

Mink JW, Thach WT (1993) Basal ganglia intrinsic circuits and their role in behavior. Curr Opin Neurobiol 3:950-957.

Monchi O, Petrides M, Petre V, Worsley K, Dagher A (2001) Wisconsin Card Sorting revisited: distinct neural circuits participating in different stages of the task identified by event-related functional magnetic resonance imaging. J Neurosci 21:7733-7741.

Owen AM, Roberts AC, Hodges JR, Summers BA, Polkey CE, Robbins TW (1993) Contrasting mechanisms of impaired attentional set-shifting in patients with frontal lobe damage or Parkinson's disease. Brain 116:1159-1175

Palencia CA, Ragozzino ME (2005) The contribution of NMDA receptors in the dorsolateral striatum to egocentric response learning. Behav Neurosci 119:953-960.

Pantelis C, Barber FZ, Barnes TR, Nelson HE, Owen AM, Robbins TW (1999) Comparison of set-shifting ability in patients with chronic schizophrenia and frontal lobe damage. Schizophr Res 37:251-270.

Paxinos G, Watson C (1998) The rat brain in stereotaxic coordinates, Ed 4. San Diego: Academic.

Pennartz CMA, Groenewegen HJ, Lopes Da Silva FH (1994) The nucleus accumbens as a complex of functionally distinct neuronal ensembles: An integration of behavioural, electrophysiological and anatomical data. Prog Neurobiol 42:719-761.

Ragozzino ME (2002) The effects of dopamine D1 receptor blockade on the prelimbic-infralimbic areas on behavioral flexibility. Learn Mem 9:18-28.

Ragozzino ME, Detrich S, Kesner RP (1999) Involvement of the prelimbicinfralimbic areas of the rodent prefrontal cortex in behavioral flexibility for place and response learning. J Neurosci 19:4585-4594.

Ragozzino ME, Ragozzino KE, Mizumori SJ, Kesner RP (2002) Role of the dorsomedial striatum in behavioral flexibility for response and visual cue discrimination learning. Behav Neurosci 116:105-115.

Reading PJ, Dunnett SB (1991) The effects of excitotoxic lesions of the nucleus accumbens on a matching to position task. Behav Brain Res 46:17-29.

Reynolds SM, Zahm DS (2005) Specificity in the projections of prefrontal and insular cortex to ventral striatopallidum and the extended amygdala. J Neurosci 25:11757-11767.

Schoenbaum G, Setlow B (2003) Lesions of nucleus accumbens disrupt learning about aversive outcomes. J Neurosci 23:9833-9841.

Setlow B, McGaugh JL (1998) Sulpiride infused into the nucleus accumbens posttraining impairs memory of spatial water maze training. Behav Neurosci 112:603-610.

Slamecka NJ (1968) A methodological analysis of shift paradigms in human discrimination learning. Psychol Bull 69:423-438.

Stefani MR, Groth K, Moghaddam B (2003) Glutamate receptors in the rat medial prefrontal cortex regulate set-shifting ability. Behav Neurosci 117:728-737.

Stern CE, Passingham RE (1995) The nucleus accumbens in monkeys ( $M a-$ caca fascicularis). III. Reversal learning. Exp Brain Res 106:239-247.

Tai CT, Cassaday HJ, Feldon J, Rawlins JN (1995) Both electrolytic and excitotoxic lesions of nucleus accumbens disrupt latent inhibition of learning in rats. Neurobiol Learn Mem 64:36-48.

van den Bos R, Charria Ortiz GA, Cools AR (1992) Injections of the NMDAantagonist D-2-amino-7-phosphonoheptanoic acid (AP-7) into the nucleus accumbens of rats enhance switching between cue-directed behaviours in a swimming test procedure. Behav Brain Res 48:165-170.

Weiner I (1990) Neural substrates of latent inhibition: the switching model. Psychol Bull 108:442-461.

Weiner I, Feldon J (1997) The switching model of latent inhibition: an update of neural substrates. Behav Brain Res 88:11-25.

Weiner I, Gal G, Rawlins JN, Feldon J (1996) Differential involvement of the shell and core subterritories of the nucleus accumbens in latent inhibition and amphetamine-induced activity. Behav Brain Res 81:123-133. 